\title{
Cooperation Dynamics on Mobile Crowd Networks of Device-to-Device Communications
}

\author{
Yong Deng, Guiyi Wei, Mande Xie, and Jun Shao \\ School of Computer Science and Information Engineering, Zhejiang Gongshang University, Hangzhou 300018, China
}

Correspondence should be addressed to Guiyi Wei; weiguiyi@gmail.com

Received 8 January 2016; Accepted 23 February 2016

Academic Editor: Tingting Chen

Copyright (C) 2016 Yong Deng et al. This is an open access article distributed under the Creative Commons Attribution License, which permits unrestricted use, distribution, and reproduction in any medium, provided the original work is properly cited.

The explosive use of smart devices enabled the emergence of collective resource sharing among mobile individuals. Mobile users need to cooperate with each other to improve the whole network's quality of service. By modeling the cooperative behaviors in a mobile crowd into an evolutionary Prisoner's dilemma game, we investigate the relationships between cooperation rate and some main influence factors, including crowd density, communication range, temptation to defect, and mobility attributes. Using evolutionary game theory, our analysis on the cooperative behaviors of mobile takes a deep insight into the cooperation promotion in a dynamical network with selfish autonomous users. The experiment results show that mobile user's features, including speed, moving probability, and reaction radius, have an obvious influence on the formation of a cooperative mobile social network. We also found some optimal status when the crowd's cooperation rate reaches the best. These findings are important if we want to establish a mobile social network with a good performance.

\section{Introduction}

With the rapid development of wireless communication technology, communications between smart devices (such as smart phones, Pads, wearable devices, and smart vehicles) for information dissemination and network resource sharing are becoming more and more common. Numerous researches have been done to solve the design challenges of wireless device-to-device (D2D) communication. Many new approaches have been proposed to make the formation of D2D communication networks possible [1-4]. As an emerging and innovative technology in next-generation cellular networks, LTE-A is capable of making the most of topology in networking its nodes. It enables wireless nodes to communicate directly with each other without support from the network infrastructure. Another enabling technology is WiFi direct, which supports devices to connect with each other for file transfer without requiring a wireless access point. Both of LTE-A and WiFi direct technologies make geography-aware information sharing and collaborative task solving within a crowd easier in our daily life.

The handheld smart devices connect with each other via direct wireless $\mathrm{D} 2 \mathrm{D}$ communication when they come across and develop the whole crowd into a self-organized network. We call such network mobile crowd network (MCN). In general, there is no central control mechanism to manage the communications between nodes in MCNs. Each node decides to participate in or get out of the network independently by itself. A node shares its resources and cooperates with other nodes voluntarily when it is in the network. Communication links between nodes are usually temporal and volatile due to nodes' movement. Nodes' mobility attributes (including moving probability, velocity, direction, and trajectory) are hard to predict; that is, the topology of a $\mathrm{MCN}$ is fully dynamic.

With resource sharing and cooperation between nodes, MCN brings us higher network coverage and bigger network capacity $[1,2]$. MCN also allows its nodes to experience additional benefits in terms of smaller communication latency, increased data rate, and reduced energy consumption under well-designed cooperation and incentive mechanism [5]. Furthermore, MCN provides a new paradigm of mobile crowd applications, such as community dynamics monitoring in public safety, traffic monitoring and planning in cities, geography-aware and participatory data collection in environment and weather sensing, and other collaborative 
complex task solving on mobile crowd, also known as mobile crowdsensing/crowdsourcing.

D2D Communications in MCNs can be treated as a type of cooperation behavior between nodes that are willing to transport or relay data for the nodes around them by some types of interdependency and reciprocity. It is the collaborative communications that significantly improve the whole network quality and throughput capacity [6]. However, there are some obstacles in promoting the cooperation behaviors that can improve the network performance of MCNs.

First, the MCN is a type of fully distributed and selforganized wireless network. In MCNs, unlike wireless ad hoc networks and wireless sensor networks, there are no network operators or coordinator nodes that work on the whole network level, have rich information of the whole network, and take charge of network performance management. Therefore, it is hard to design and deploy a centralized control mechanism for improving the collaborative communications in MCNs.

Second, most nodes are usually handheld smart devices. The resource sharing at application level or system level may be turned on/off any time anywhere by the device holders. The collaborative communications between these autonomous nodes fully depend on human-centric communication will. Due to the fact that all the mobile devices are carried by different people who are irrelevant, a natural problem is whether the device holders are willing to cooperate when they communicate with their neighbors. As we know, altruistic behaviors in D2D communications, that is, sharing one's own resources with others, induce extra energy consumption and utility loss in storage, computing, and communication. Therefore, it is reasonable that MCN nodes tend to make selfish decisions without effective incentive mechanisms; that is, they do not share their resources with others. Selfish behaviors will destroy the attempt of cooperation promotion in MCNs. Therefore, the incentive mechanisms designed for data-centric self-organized networks, such as mobile ad hoc networks and vehicular networks, can not work effectively in the participatory human-centric networks like MCNs, due to possible frequent human intervention.

Third, mobility attributes of nodes, such as moving possibility, velocity, and direction, also substantially affect the cooperation between nodes. Mobility attributes affect the meeting possibility and communication connectivity between nodes. Ephemeral linkages between nodes make the topology of a MCN highly variable. Thus, topology-based incentive mechanisms can not be directly used to promote the cooperation in MCNs, such as tit-for-tat, reputation schemes, and auction scheme $[7,8]$ proposed for structured networks. Before designing an effective incentive mechanism for MCNs, we should know how nodes' mobility attributes affect the resource sharing behaviors (cooperation or defection) in a MCN. However, to the best of our knowledge, there is still no comprehensive work on MCNs that investigate the relationship between the nodes' mobility and the cooperation rate of a MCN.

In this paper, our objective is not to design an effective incentive mechanism to promote the cooperation rate of a specified MCN but to explore the cooperation dynamics on generalized MCNs, to investigate the factors that influence the cooperation rate of MCNs, and, more importantly, to analyze the relationships between the cooperation rate and the main influence factors in MCNs. To tackle the above obstacles, we classify the main influence factors on the cooperation rate of MCNs into three categories as below.

(i) Device Factor. The device factor is double-edged [9]. The advantageous aspect of device factor is that a device may benefit from the cooperation with other nodes, such as increasing network performance, enlarging its sensing coverage, and promoting application specific performance. However, the main disadvantageous aspect of device factor is the supernumerary resource consumption incurred by altruistic resource sharing in cooperation, including energy consumption, bandwidth loss, additional CPU cycles, and memory occupation.

(ii) Network Factor. The network factor mainly comes from nodes' mobility, including moving probability, moving speed, and direction changing. Suppose each smart device can communicate with multiple neighbored nodes, simultaneously (in this paper, we analyze D2D communication in the application layer. We consider a node can communicate with multiple other nodes, simultaneously; the implementation of multiple-to-multiple D2D communication in the physical layer is not the focus of this paper). The length of wireless communication link (or radius) and the density of the nodes (or population) in a network also affect the cooperation rate in MCNs.

(iii) Human Will. The smart device holders make the final decision, sharing or not. Suppose all participants in a MCN are rational. Therefore, they all make cost-efficient decisions. As a result, we always need appropriate rewards to incentivize more cooperative participants [10]; thus, we can build a satisfying MSN. According to the most prestigious work on social behaviors, such as $[7,8,11-13]$, reciprocity mechanism leads to high cooperation rate in social networks. These works show that incentive and punishment play important roles in cooperation behaviors.

\section{Contribution}

With considerations of three influence factors and possible social dilemma mentioned in Section 1, our objective is to find out how and to what extent the cooperation rate is influenced and changes. Without loss of generality, we study the cooperation dynamics on the mobile crowds of unstructured population that locate, move, and communicate with each other within a fixed-size region. To highlight the influence from nodes' mobility, under the same population size and region size, we compare the cooperation dynamics on mobile crowds with that on the crowds of static spatial structures. Furthermore, to analyze the situation of more reality, we also explore the cooperation dynamics on the MCN that contains a proportion of special nodes, which is designedly placed into the crowd to promote or degrade the cooperation rate of the MCN. In this paper, we use Prisoner's 
Dilemma Game (PDG) model to feature the cooperation behaviors in MCNs. Then, from an evolutionary game theoretic perspective, we construct an evolutionary PDG model to explore the relationships between the cooperation rate and the main influence factors on it. The proposed game model takes full account of the three types of influence factors. We think the quantitative findings are valuable for designing effective incentive mechanisms in MCNs.

Our main contributions in this work are as follows:

(i) We model the cooperation dynamics on MCNs of D2D communications into an evolutionary dynamic PDG model, in which smart devices are treated as rational game players/agents. To the best of our knowledge, this is a novel work that analyzes the cooperation dynamics on MCNs taking into consideration the above three categories of influence factors simultaneously. In our model, the device factor and the human will are featured by the parameterised payoff function and evolutionary rule. The agents (handheld smart devices) are supposed to be selfish and rational. Meanwhile, they also expect to construct reciprocity relationships with other agents. Therefore, the network factor is mainly featured by dynamic network topology and agents' mobility attributes. Comprehensive experiments and simulations on the unstructured crowd population show the cooperation rate of MCNs presents no-trivial changing when the influence factors change by time. The experimental results also quantitatively indicate the relationships between the cooperation rate and the main influence factors, including density of agents, communication range, temptation to defect, and mobility attributes.

(ii) We fully investigate the impact on the cooperation rate of MCNs from agents' mobility. The mobility attributes we mainly focus on in this paper include moving probability and velocity. Consider that the nodes in the MCN do not change their places all the time. We novelly introduce moving probability which can be viewed as the stability of the crowd and then systematically investigate the cooperation rate under different moving probability, speed, communication radius, and temptation to defect. We find too fast and too slow moving speed both lead to low cooperation rate by fixing other parameters. For a given crowd, it can be observed that there is an optimal average moving speed to achieve the highest cooperation rate. Furthermore, for a given average moving speed, the crowd's cooperation rate increases when the average moving probability increases from static situation; and the cooperation rate decreases when the average moving probability continuously increases from a certain average moving probability. There is an optimal moving probability for achieving the highest cooperation rate.

(iii) Based on our experiment results, we find that the cooperation rate of a mobile crowd is low when the crowd population is both too scattered and too dense. And the highest cooperation level is achieved with a moderate crowd density. We also find that the highest cooperation rate occurs under agent's moderate communication radius. In particular, when agents' communication radius is larger or smaller than the moderate value range of the given configuration, the cooperation rate of the mobile crowd declines significantly.

(iv) Simulations on the square plane indicate that the cooperation rate on a mobile crowd has no-trivial dependence on the individuals behaviors including moving probability, speed, communication radius, and temptation to defect.

\section{Related Work}

The formation of cooperative D2D communication between the mobile nodes is of great importance to an efficient cooperative wireless network, in which individuals share data with their neighbors to substantially boost the whole network performance. Numerous researches have been done to solve the design challenges of a D2D network, and many new approaches have been proposed to make the formation of a D2D network possible $[1,3,4]$.

Nevertheless, except for the design challenges, since the fact that all the mobile devices are carried by different people who are irrelevant, a natural problem is whether the device holders are willing to cooperate when they communicate with their neighbors [14].

Given the fact of explosive growth of online social networks such as Facebook, Twitter, and Weibo, some researches novelly leverage the social relationship to promote a cooperative D2D network [5]. There are also some papers trying to investigate the cooperation rate in a Public Goods Game (HPGG) and Prisoner's Dilemma Game (PDG) modeled collaborative social networks (CSNs) $[15,16]$.

As we know, with the popularity of D2D likely communication model, more and more crowdsensing and crowdsourcing applications will be deployed in the flocking population. The realization of such apps is deeply dependent on the cooperation of the mobile nodes. Most existing works place their nodes on a given spatial structure and demonstrate appearance of cooperative nodes in such spatial structure from the view of evolutionary mechanism [17-19].

Recently, research about the statistical properties of human motion has attracted much attention. Compared with agents on some specific network topology, we think this meaningful extension is natural and more close to our real social network, given the fact that motion is a fundamental property of individuals and agents may change their place in a typical crowdsensing or crowdsourcing environment. Nevertheless, most researches about this just assume that each node will move all the time during the simulation [20$22]$. In our paper, we assume that the nodes locate on a square plane and each node may move with a probability.

Prisoner's Dilemma (PD) game has long been one of the most popular game models to simulate the biological systems and human behavior [11, 21]. Like most researches about the CSN applications, we also use the PDG to model 
the cooperation D2D dynamics on the networks. In a PDG modeled D2D communication, each node can choose to cooperate or defect simultaneously and then get their payoff. When a node is willing to sacrifice itself to transfer data for its neighbors, we say that it chooses to cooperate, and we call it a cooperator; otherwise, we consider it a defector. A defector means someone who only gets the data they need from the others and sacrifice nothing to help the others.

Such social dilemma is commonly observed throughout the human societies. The emergence and existence of cooperation remain an open question in biology and social science [12]. Tones of works have been done to study the showing and persistence of cooperation in a population composed of selfish individuals [23-25]. In a broad range of disciplines, evolutionary game theory is becoming one of the most fruitful frameworks to investigate this dilemma.

Since Nowak and May have first shown us that the evolutionary PD on a simple spatial structure can induce remarkable cooperation emergence [13], numerous researches have been done to understand the interplay between network topology and evolutionary game. Some natural behaviors in the real world have also been introduced at the same time and many interesting phenomena have been observed. We have already known that social relationship such as kin selection, direct reciprocity, indirect reciprocity, network reciprocity, and group selection has some potential to lead to cooperation [7]. In order to stimulate cooperation among D2D communications, some researches try to leverage human social relationship to solve the cooperation problem in the collaborating D2D network $[5,26]$. Recently, research about the statistical properties of human motion has attracted much attention. Recently, many researches extended the research on evolutionary games to the systems consisting of mobile agents which are randomly located on a square plane and play games with agents around them [11].

\section{Mobile Crowd Network Model}

The mobile crowd network we study in this paper was formed by some moving nodes. The nodes meet, connect, and share resources with each other opportunistically. Each node is an autonomous and rational agent in the cooperations with others. Many features of the nodes affect their cooperation behaviors, mainly including crowd density, moving probability, speed, communication radius, and temptation to defect. For the easy understanding of the MCN model, we list some important notions as follows:

$N$ : the number of players in a game.

$L$ : the length of the square plane.

$\rho$ : the density of the crowd.

$v_{i}(t)$ : node $i$ 's moving speed at moment $t$.

$\theta_{i}(t)$ : node $i$ 's moving direction at moment $t$.

$f_{c}$ : crowd's cooperation rate.

$p$ : moving probability.

$v$ : moving speed. $\operatorname{pos}_{i}(t)$ : node $i$ 's position in round $t$.

$\operatorname{pof}_{i}(t)$ : node $i$ 's payoff in round $t$.

$s_{i}(t)$ : node $i$ 's strategy in round $t$.

$k_{i}(t)$ : the number of neighbors of $i$ at round $t$.

$b$ : temptation to defect.

$r$ : reaction radius.

4.1. Mobile Crowd Network. Suppose a mobile crowd consists of $N$ nodes (or smart devices) moving within a $L \times L$ square plane with periodic boundary conditions. Initially, the $N$ nodes randomly locate on the plane and randomly move. They meet and communicate with each other via D2D communication for reciprocal resource sharing. Here, two nodes meet means they both locate inside the other's communication coverage range. The momentary connections between the $N$ nodes make them dynamically form a mobile crowd network, MCN.

Here, we use $\rho=N /(L \times L)$ to represent the density of the crowd. In the MCN, each node $i(i \in[1, \ldots, N])$ has a fixed identical communication radius $r$. It is indicated that the mean degree of all nodes is $\rho \pi r^{2}$. We use $v_{i}(t)$, and $\theta_{i} \epsilon$ $[0,2 \pi)$ denotes $i$ 's moving speed and direction at moment $t$, respectively. At any time, $i$ can just contact its neighbor nodes, that is, the set of nodes locating within $i$ 's communication coverage. At time $t$, $i$ 's neighbor set is denoted as $w_{i}(t)$, where $w_{i}(t)=\left\{j \mid E(i, j)<r_{i}, j \in N, j \neq i\right\}$. Here, $E(i, j)$ represents the Euclidean distance between $i$ and $j$.

In this $\mathrm{MCN}$, the cooperation behaviors between nodes are constrained by nodes' D2D communication radius and their cooperation willingness. When $j \in w_{i}(t) \wedge i \notin w_{j}(t)$, we assume that $i$ can just transfer data to $j$ and $j$ can not receive data from $i$. This means $i$ and $j$ form a unidirectional communication. When $j \in w_{i}(t) \wedge i \in w_{j}(t), i$ and $j$ can transfer data to and receive data from each other based on their cooperation willingness, which is a bidirectional communication.

4.2. Prisoner's Dilemma Game on MCNs. The cooperation behaviors on the MCN described above can be modeled by a Prisoner's Dilemma Game (PDG), in which the $N$ independent nodes are agents of the game. Each node plays PDG with its neighbors round by round with fixed period of time for a round when any two nodes meet each other. All nodes synchronously choose their strategies and compute their payoffs in a round.

A node autonomously makes decision of cooperation or defection that means sharing or not sharing resource to its neighbor nodes. Each node can just choose its strategy: cooperate $(C)$ or defect $(D)$. $C$ strategy means sharing while $D$ means not sharing. A node with a strategy $C$ is a cooperator; otherwise, it is a defector. A cooperator will have to sacrifice its own resources; as a consequence, cooperator will create a benefit to each of its neighbors with its own resource consumption. On the contrary, a defector will not lose anything but only benefit from its cooperative neighbors. The

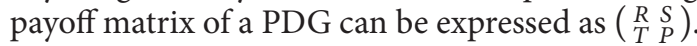


Two nodes meet and both get a payoff $R$ for mutual cooperate and $P$ for mutual defect. When two cooperators meet together, they will choose to sacrifice themselves to benefit each other, so they get the same payoff $R$ equal to their benefits from cooperative neighbors minus their consumption by choosing to be a cooperator. However, two defectors will do nothing for each other and get no reception from each other, which means that their payoff $P=0$. Things will change when a cooperator meets a defector; cooperator will choose to sacrifice itself to share its information with the defector, yet the defector will do nothing but only get the benefits from its cooperator neighbors. Thus, cooperator's payoff by playing with a defective neighbor $S$ will be less than 0 , because it will sacrifice its resources but get nothing from the defector; on the other hand, the defector's payoff $T$ will be the largest, because it gets what it needs without sacrificing anything. It is easy to deduce that $T>R>P>S$ for all nodes. Therefore, defector always outperforms the cooperator since it will get more no matter what its neighbor is. However, when all nodes choose $D$ strategy, they will all get nothing from others and the network is out of action. The situation that the whole network suffers from the problem of low cooperation rate is called social dilemma (the Prisoner's Dilemma Game model first illustrates the conflict of interests between what is best for the individual and what is best for the group and creates the social dilemma [27]).

To investigate the cooperation dynamics on MCNs, we model the long-term interactions among mobile nodes into an evolutionary PDG. At the beginning of the evolution process, each node randomly chooses its strategy $(C$ or $D)$ at its initial location. Each agent plays a PDG with their neighbors synchronously. In this multiround (or multigeneration) evolutionary game, one round is divided into two phases. At the first phase, all nodes compute and get payoffs according to their strategies. At the second phase, all nodes move and choose their next round strategies. Since a node locates at different position and meets different neighbors, its strategy changes in different round. It is obvious that the multiround game is different from iterated PDGs, since a node's game opponents are changing in different rounds.

By adopting the common practice of the configuration in evolutionary PDGs, such as the work $[11,21,22]$, we let $T=b$, $R=1$, and $P=S=0$ in the payoff matrix. $T=b$ can be considered as the temptation to defect, $1<b<2$. Thus, we rescale the payoff matrix as $\left(\begin{array}{ll}1 & 0 \\ b & 0\end{array}\right)$.

In the interval from round $t$ to $t+1$, agent $i$ moves with velocity $v_{i}(t)$ and direction $\theta_{i}(t)$. Here, $\theta_{i}(t)$ is a variable randomly chosen at the second phase of round $t, \theta_{i}(t) \in[0,2 \pi)$. This means that topology of the MCN will change irregularly. Different from most related work in mobile crowd/social networks, we novelly introduce a new parameter $p$ to describe a node's moving probability realistically. When all nodes obtained their payoffs in round $t$, each node should choose its strategy for the next round $t+1$. Let $\operatorname{pof}_{i}(t)$ denote node $i$ 's payoff and $s_{i}(t)$ denote $i$ 's strategy in round $t$. In this evolutionary game, a node changes its strategy according to the following mechanism:

(1) Agent $i$ does not need to change its strategy when $i$ has no neighbor node in round $t$; that is, $s_{i}(t+1)=s_{i}(t)$.
(2) Agent $i$ randomly chooses a neighbor $j$ and compares $\operatorname{pof}_{i}(t)$ with $\operatorname{pof}_{j}(t)$. If $\operatorname{pof}_{i}(t)>\operatorname{pof}_{j}(t), i$ will keep its strategy not changing in the next round; that is, $s_{i}(t+1)=s_{i}(t)$.

(3) Agent $i$ randomly chooses a neighbor $j$ and compares $\operatorname{pof}_{i}(t)$ with $\operatorname{pof}_{j}(t)$. If $\operatorname{pof}_{i}(t) \leq \operatorname{pof}_{j}(t), i$ adopts $j$ 's $t$ round strategy, that is, $s_{i}(t+1)=s_{j}(t)$, with a probability $\left(\operatorname{pof}_{j}(t)-\right.$ $\left.\operatorname{pof}_{i}(t)\right) /\left(\max \left\{k_{j}(t), k_{i}(t)\right\} \cdot b\right)$. The probability is proportional to the payoff difference [22]. Here, $k_{i}(t)$ and $k_{j}(t)$ are the number of neighbors of $i$ and $j$ at round $t$.

It is notable that this mechanism is important for the multiround evolution process because a rational agent tends to change its strategy when it is not satisfied with its payoff in previous round. All agents carry out the strategy update process synchronously. In round $t$, the cooperation rate of the MCN is $f_{c}$, where $f_{c}=N_{C} / N$; here $N_{C}=\operatorname{count}\left\{i \mid p_{i}(t)=C\right\}$ for $i=1, \ldots, N$.

\section{Experiments and Discussions}

In this section, Using evolutionary PDG model, we carry out simulations on the MCN to investigate the cooperation rate changing by the moving probability $(p)$, speed $(v)$, radius $(r)$, and temptation to defect $(b)$. In our experiments, the MCN consists of 1000 mobile nodes. The crowd move on a $50 \times 50$ square. To avoid the border effects, we assume the square plane is toroidal. Under a given $p$, we give agents' radius different distributions. We also compare the cooperation dynamics of the mobile crowd with different radius distributions, including uniform, normal, exponential, and power-law. In order to figure out the influence of the others, we configured the other three parameters as identical features for all agents, and then changed them accordingly to get different cooperation dynamics.

Suppose the position of agent $i$ at time (or round) $t$ is $\operatorname{pos}_{i}(t)=\left(x_{i}(t), y_{i}(t)\right)$. At round $t, v_{i}(t)$ refers to agent $i$ 's moving speed and $\theta_{i}(t)$ means agent $i$ 's moving direction. Then, $i$ 's position at next round $t+1$ is $\operatorname{pos}_{i}(t+1)=\left(x_{i}(t+\right.$ $\left.1), y_{i}(t+1)\right)$ which can be easily computed on a toroidal surface. To simplify the computing, we let time interval from round $t$ to $t+1$ be 1 .

In the simulations, to guarantee accuracy, we collect data traces of the last 3,000 generations (rounds) from 50,000 generations. And each piece of data is an average of 100 runs under the same configuration.

5.1. Moving Probability with Different Moving Velocity. Moving probability determines the stability of the nodes and affects the cooperation between agents. To analyze the affection on cooperation rate $\left(f_{c}\right)$ from agents' moving probabilities $(p)$, in the experiment, we calculate the $f_{c}$ by changing agents' moving probability $p$ and fixing the other parameters, including velocity $(v)$, radius $(r)$, crowd density $(\rho)$, and the temptation to defect $(b)$. The result of this experiment is shown as Figure 1.

Compared with related work [11] which demonstrated that the cooperation rate can be enhanced under a moderate value of $b$ and $v$, in Figure 1, we find the cooperation rate of the whole population is also greatly improved with a moderate 


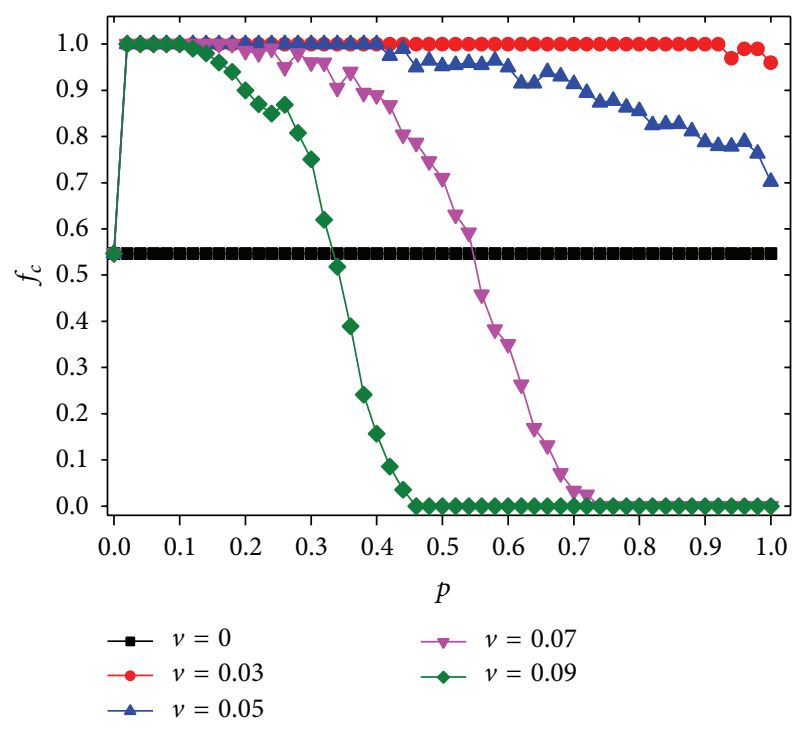

FIgURE 1: (Color online) The cooperation rate $\left(f_{c}\right)$ under different agents' moving probability $p$ and velocity $(v)$. Here, we fix $L=50$, $\rho=1.3, b=1.1$, and $r=1$. The black line means the crowd is static $(v=0)$.

$p$ and $v$. Compared with the static network model $(v=0)$ denoted by the black line in Figure 1, when letting crowd nodes move with some moderate probability, cooperation rate improves significantly in the crowd.

It is obvious that the cooperation rate decreases monotonously with $p$ increasing, but is irrelevant to the changing of $v$. When $p$ is fixed, we find that $f_{c}$ is also decreasing while $v$ is increasing. It is notable that the decline curve of different velocity ( $v=0.03, v=0.05, v=0.07$, and $v=0.09$ ) has different amplitude. When $v$ is equal to 0.03 , $f_{c}$ remains almost the same and only a tiny decrease can be observed when $p$ is increasing to almost 1 . This means the influence of $p$ is limited with a moderate $v$. But when $v$ comes to the larger situation, the influence of $p$ appears, which is demonstrated by the curves of $v=0.05, v=0.07$, and $v=0.09$. Looking at these curves, it can be found that the decline extent of $f_{c}$ becomes quicker when agents' moving speed increases. And the dominance of mobility can be only observed in a relatively smaller region of $p$. Based on this discovery, we find that the influence of $p$ is also partly determined by agents' moving speed. The influence on cooperation rate from agents' moving probability is more significant under a relatively larger moving speed.

In Figure 1, we also realize that when the whole mobile crowd move fast, a perfect cooperation network can be formed with a moderate moving probability, which means that individuals of the crowd are relatively stable. On the other hand, when the speed of the crowd is relatively slow, the cooperation rate achieves a high level even though the crowd is not stable enough, which means that the moving probability has relatively larger impact on cooperation rate than moving speed.

5.2. Crowd Evolutionary Dynamics. Capturing a series of snapshots from the evolutionary process, we find some major evolution characteristics of the mobile crowd network system. Figure 2 shows the snapshots at eight times for a simulation with $v=0.07$, which indicates how a mobile crowd evolve to a full cooperation network with some favorable conditions. At time 0 [Figure 2(a)], both cooperator [green dots] and defector [red dots] players are randomly located on the squared plane, with the same fraction (0.5). Because agents move with a probability, the number of connections for each player will change. Thus, it is essentially different from the static status. Not too long after the initial state, Figure 2(b) shows the state of the system at $t=100$. We can see a quick decrease of the cooperators. Most individuals turn to a defector for a better payoff, except for a small cluster of cooperators on the top right corner of the square.

Many existing related works had revealed that the cooperators can enforce their success only by forming clusters in a mobile environment [21]. In our simulation, one can also find that the small cooperator cluster becomes expanding as time grows. From Figures $2(\mathrm{c})-2(\mathrm{~h})$, we can see the cooperators slowly expand to the whole square based on the small cooperator region. Then, at about 3000 time steps after $t=20000$, the system finally evolves to a cooperative network.

Figure 2 has shown how cooperators form clusters and then attract the defect nodes that are lying around the boundary to ensure the success of cooperation. In order to qualitatively explain the results generated in Figure 1, we evaluate the mean payoffs of cooperators and defectors lying around the boundary when $v=0.07$. Because all nodes are rational, their strategies will change according to how much payoff they can receive. As Figure 3 shows, the average payoff of the defectors $\left(P_{d}\right)$ around the boundary is 0 when the moving probability $(p)$ is less than 0.38 ; on the contrary, the payoffs of the cooperators $\left(P_{c}\right)$ on the boundary are at the highest level. So, at this region of $p$, we can know that defectors have no place to live $\left(f_{c}=0\right)$, while cooperators flourish in the crowd and get their highest payoff. When $p$ is bigger than $0.38, P_{c}$ begins to decrease and $P_{d}$ begins to increase. We are able to see the decreasing of cooperators and the defectors appear and keep increasing. Interestingly, when $p$ is more than 0.62 , both $P_{c}$ and $P_{d}$ tend to decline. This may be owing to the lasting growth of the defectors. When the number of defectors is big enough, then defectors will have more chances to communicate with the ones that are also defectors. Compared with the case that most nodes lying around the defective one are cooperator, as a matter of fact, this will definitely lower their payoffs. Then, at $p=$ 0.74 , we can find both cooperators and defectors get a payoff of 0 , which means the crowd falls into a defection state, and defectors can never gain payoffs by exploiting their cooperator neighbors.

5.3. Moving Probability under Various Defect Temptation. In order to analyze the role of moving probability, given the condition that the temptation to defect $(b)$ is changing, Figure 4 shows us the cooperation level curves of our simulation results for $\left(f_{c}\right)$ as a function of the temptation to defect $b$. Each curve indicates different moving probabilities $(p)$, here 


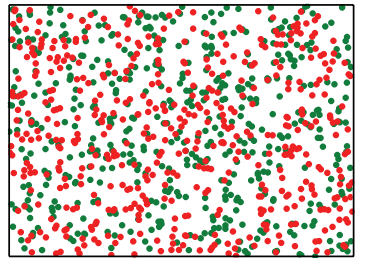

(a) $t=0$

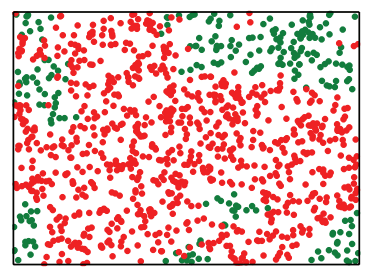

(e) $t=5000$

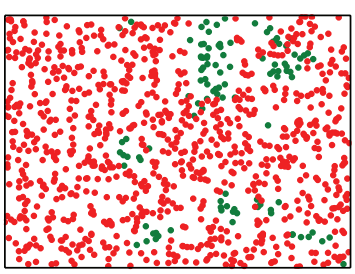

(b) $t=100$

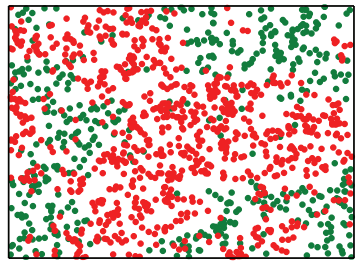

(f) $t=7000$

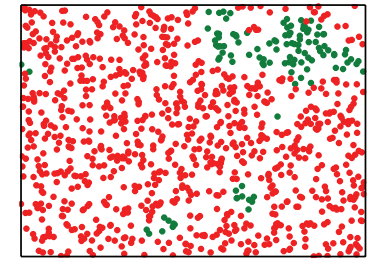

(c) $t=1000$

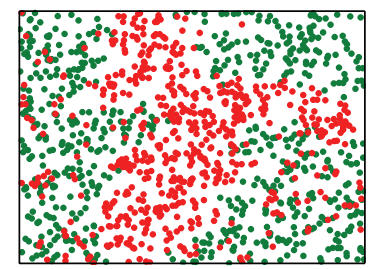

(g) $t=10000$

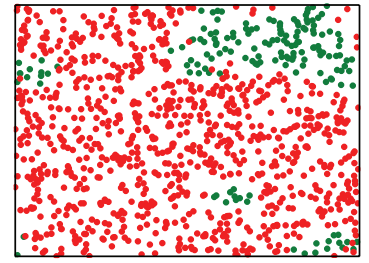

(d) $t=3000$

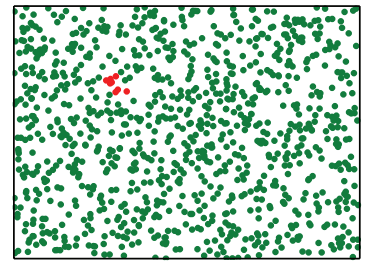

(h) $t=20000$

FIgURE 2: (Color online) Spatiotemporal evolution of a mobile crowd (each agent moves with a probability) where $p=0.1$. This specific simulation has been performed for a population in which $N=1000, \rho=1.3, b=1.1$, and $v=0.03$. Cooperators are denoted by green (light gray) and defectors are denoted by red (dark gray) dots correspondingly. Each picture depicts a snapshot of different time of evolution process.

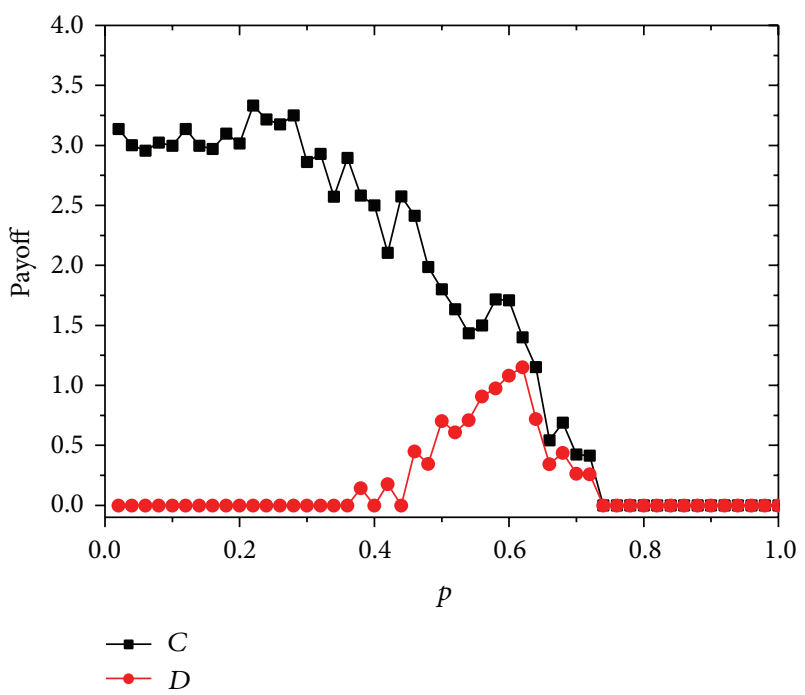

Figure 3: The mean payoffs of cooperators and defectors who are lying around the boundary. Here, we fix $L=50, \rho=1.3, b=1.1$, $r=1$, and $v=0.07$

we have four curves indicated as $p=0, p=0.1, p=$ 0.5 , and $p=0.9$. Not surprising, like most classic studies about the relationship between defect temptation and $f_{c}$ in the evolutionary game theory, it is notable that $f_{c}$ decreases monotonously with $b$ increasing up to a threshold where cooperation vanishes, in both static and dynamic status. This is reasonable because larger $b$ means stronger temptation to defect. All nodes of the crowd will tend to defect when the temptation is big enough. But we can find various threshold under different $p$. When $p$ is not 0 , it is clear that the cooperation level decreases while the moving probability increases. By fixing $b$, we find that $f_{c}$ is inversely proportional to the moving probability $(p)$. When $b$ is relatively small (less

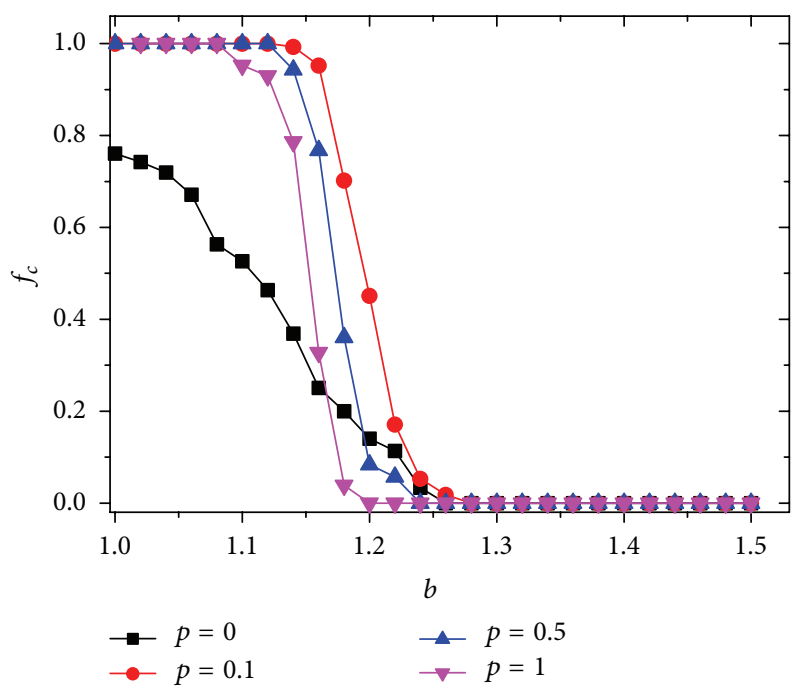

Figure 4: The relationship between the cooperator level $\left(f_{c}\right)$ and temptation to defect $(b)$ under various values of $p$. Black line indicates a static crowd $(p=0)$. Here, we set $N=1000, \rho=1.3$, and $v=0.03$.

than about 1.3), $f_{c}$ is dramatically promoted when individuals choose to move with some probability. As we see in Figure 3, $f_{c}$ for $p=0.1$ always dominates $f_{c}$ for the static status $(p=0)$. But, for a higher moving probability $(p=0.5, p=0.9)$, compared with the static status, cooperation rate is promoted in a relatively smaller region of $b$. It is about $0-1.19$ when $p=0.5$ and about $0-1.16$ when $p=0.9$.

From Figure 4, we find that when given a moderate temptation to defect, a relatively stable ( $p$ is small) crowd helps to form a cooperation network. We can also find that $f_{c}$ declines when the mobile crowd system becomes more erratic. It is also important to notice that a relatively small $p$ 


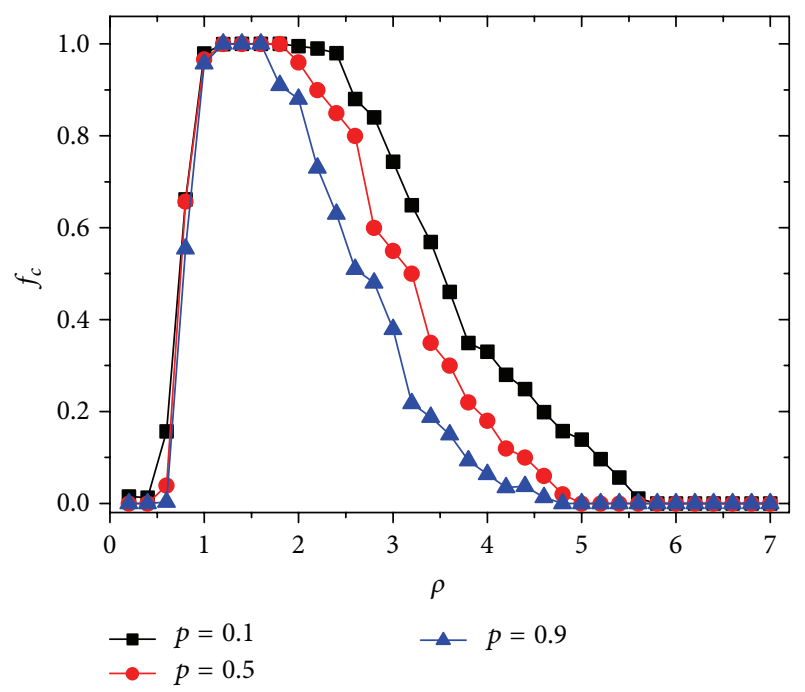

Figure 5: (Color online) The cooperator frequency $\left(f_{c}\right)$ versus crowd density $(\rho)$ under different moving probability $(p)$. Here, we fix $L=50, v=0.03, b=1.1$, and $r=1$.

means a larger region of $b$ that can make sure the cooperation is successful.

5.4. Crowd Density and Communication Radius. Similar to most related work, it is interesting to investigate the effects of $\rho$ (crowd density), $r$ (node's communication radius), and $v$ (moving speed), respectively, on the evolution of cooperation after we give the node a probability to move.

With fixed $r$ and $v$, we investigate the effect of $\rho$ on three different moving probabilities $(p)$ in Figure 5. In order to get various $\rho$, we change the crowd population $N$. Obviously, smaller $N$ means lower crowd density. Compared with the work $[11,21,25]$, we find an ideal region of the density of the players which is favorable to form a cooperation crowd under three different $p$. From Figure 4, we can see that the cooperation level $\left(f_{c}\right)$ tends to be low when the population is both too spread ( $\rho$ is small) and too dense ( $\rho$ is large) in three different $p$. For this reason, these two conditions are hard for cooperators to form clusters, the only mechanism that can enforce their success. When it comes to different $p$, the figure shows that different $p$ obtains a different ideal density region. Obviously, $p=0.1$ gets a biggest range of ideal density area that is good for the evolution of cooperation. Interestingly, when fixing $\rho$, smaller moving probability $(p=0.1)$ also dominates the larger ones $(p=0.5$ and $p=0.9)$. It indicates that a relatively smaller individual's moving probability can get a more cooperative network. At the same time, when $\rho$ is too large or small, the crowd can only get a full defective result, which means $f_{c}$ is equal to 0 .

Each node only communicates with the nodes within its radius, which means node's radius $(r)$ has an important role in the evolutionary game dynamic. Figure 6 shows mobile crowd's cooperation dynamics with four different view radii when $b$ is changing. It is obvious that $f_{c}$ monotonically decreases with the increase of $b$. However, different radius distributions can induce different cooperation levels. And we

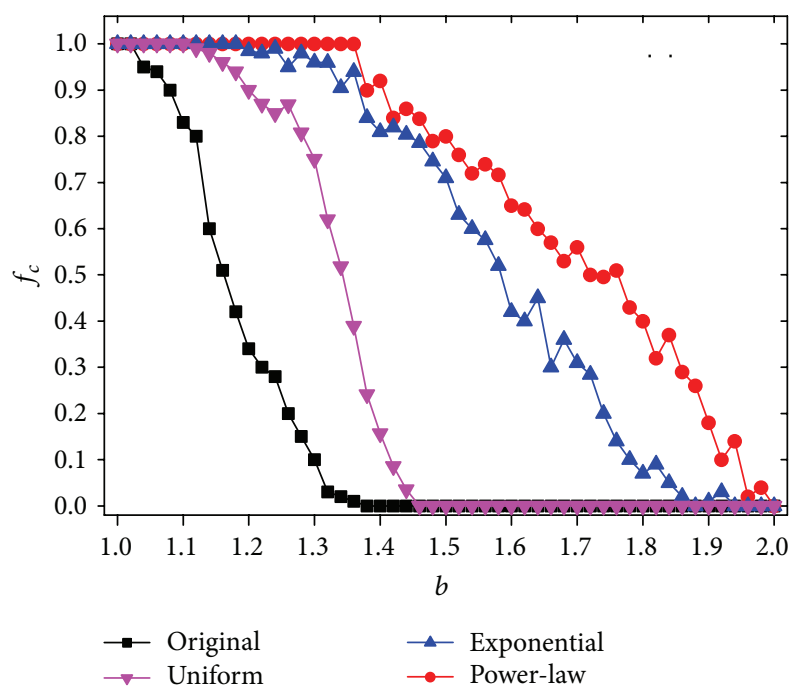

FIGURE 6: The cooperator frequency $\left(f_{c}\right)$ versus $r$ with different distributions. Here, we fix $L=50, \rho=1.3, v=0.03$, and $p=0.5$.

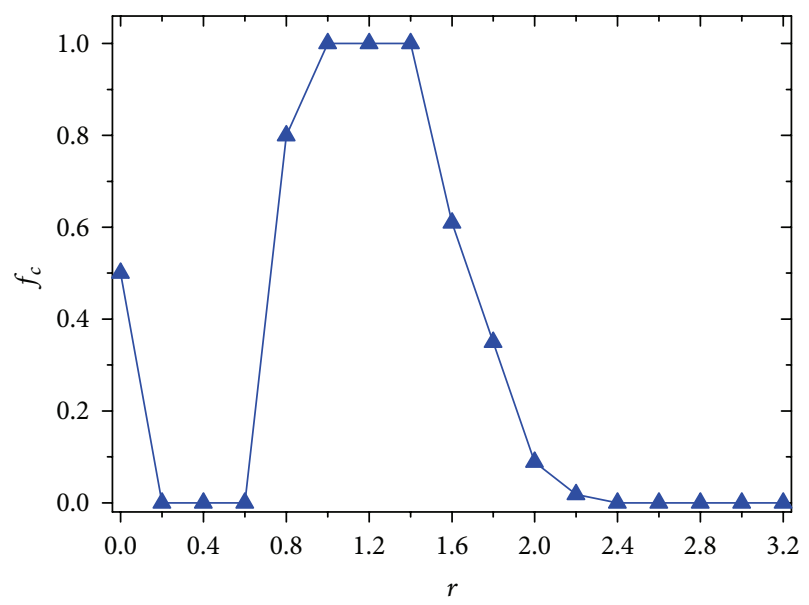

FIGURE 7: The cooperator frequency $\left(f_{c}\right)$ versus $r$. Here, we fix $L=$ $50, \rho=1.3, b=1.1, v=0.03$, and $p=0.5$.

can sort it out easily from Figure 6. We can know that crowd will get a lowest cooperation level when all the nodes have the same radius. However, the cooperation rate will be improved remarkably when the crowd has a more heterogeneous distribution. We can see the power-law distribution, which is the most heterogeneous one, gets the highest cooperation level. Previous studies have revealed that radii have some nontrivial connection with nodes' moving property [21]. From Figure 6, we infer that the influence of different radius distribution still exists under a moving probability $p$.

In Figure 7, we fix other parameters in order to investigate the effect of the view radius changing. We can find that the highest cooperator frequency can be obtained under some moderate values of $r$ ( $r$ is within 1.0-1.6). When $r$ is larger or smaller than that value region, we observe an apparent decline of the crowd's cooperation level. This is in accordance with $[21,22]$. Evidently, nodes have fewer interaction neighbors with a smaller $r$, and cooperators 


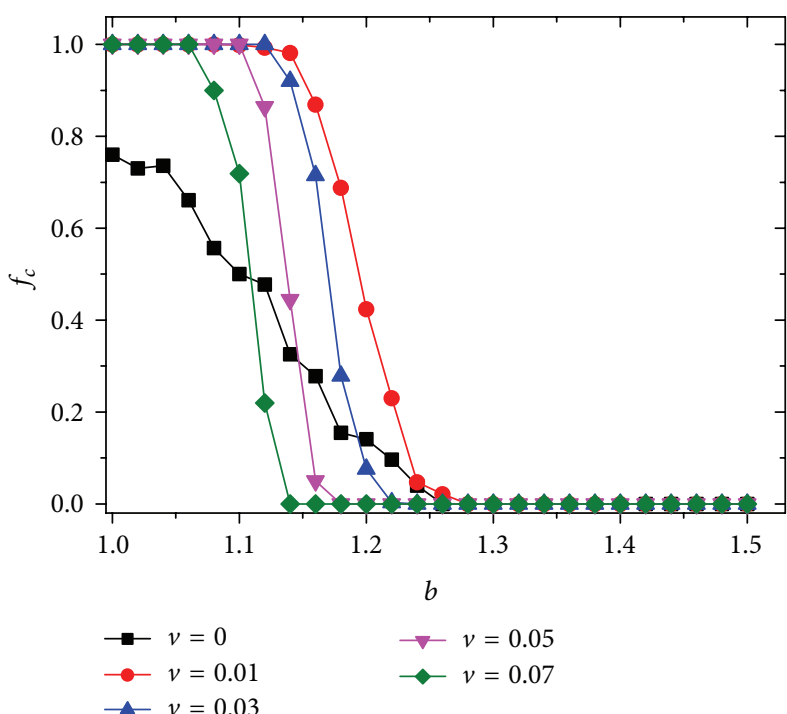

FIGURE 8: The relationship between the cooperator level $\left(f_{c}\right)$ and temptation to defect $(b)$ under various values of $v$. Black line indicates a static crowd $(p=0)$. Here, we set $N=1000, \rho=1.3$, and $p=0.5$.

cannot form clusters to resist the invasion of defectors. On the contrary, with a large $r$, nodes of the crowd tend to be mixed enough; more or less everyone interacts with each other, so we inevitably have a low cooperation level.

5.5. Moving Speed. Next, assume node will move with a probability $(p=0.5)$, we fix other parameters to investigate the effect of the velocity $v$ in Figure 8 . As in Figure 4 even with different speed, cooperation level declines when we increase the temptation of defect $(b)$. Compared with the static case $(v=0, p=0)$, it is worth noting that cooperation is greatly enhanced when players are allowed to move with a low velocity (e.g., $v=0.01$ ). Besides, different moving speed can induce different cooperation levels, and one can easily sort out the cooperation levels: $v=0.01>v=0.03>v=$ $0.05>v=0.07$. Similar to Figure 4, we also observe that a low velocity can improve the cooperator in a larger region of $b$, indicating that lower velocity can tend to be of more benefit to the survival of cooperator.

\section{Conclusion}

In this paper, we study the cooperation dynamics on a typical mobile crowd network of D2D communication. Using evolutionary game theory, our simulation and analysis on the cooperative behaviors of mobile users take a deep insight into the cooperation promotion in such a dynamical network with selfish autonomous users. The experiment results show that mobile user's features, including speed, moving probability, and reaction radius, have an obvious influence on the formation of a cooperative MCN. We also found some optimal status when the crowd's cooperation rate reaches the best. (1) The crowd can reach a good cooperation rate with a moderate moving speed and probability, which is no more than $0.5 ;(2)$ we found the best reaction radius of a node in $\mathrm{MCN}$, which is about 0.8 to 1.5 in our simulation; (3) the ideal crowd is also shown in our experiment, which is about 1 to 3 ; (4) the crowd can research a higher cooperation rate when the temptation to defect is higher, which is reasonable. These regularities are useful for a network designer to design a MCN with good performance.

\section{Competing Interests}

The authors declare that they have no competing interests.

\section{Acknowledgments}

This work was supported in part by China NSF Grants 61472365, 61472364, and 61572435 and ZJNSF Grant LZ16F020001.

\section{References}

[1] G. Fodor, E. Dahlman, G. Mildh et al., "Design aspects of network assisted device-to-device communications," IEEE Communications Magazine, vol. 50, no. 3, pp. 170-177, 2012.

[2] A. Antonopoulos, E. Kartsakli, and C. Verikoukis, "Game theoretic D2D content dissemination in $4 \mathrm{G}$ cellular networks," IEEE Communications Magazine, vol. 52, no. 6, pp. 125-132, 2014.

[3] S. Shalmashi and S. Ben Slimane, "Cooperative device-to-device communications in the downlink of cellular networks," in Proceedings of the IEEE Wireless Communications and Networking Conference (WCNC '14), pp. 2265-2270, Istanbul, Turkey, April 2014.

[4] L. Lei, Z. Zhong, C. Lin, and X. Shen, "Operator controlled device-to-device communications in LTE-advanced networks," IEEE Wireless Communications, vol. 19, no. 3, pp. 96-104, 2012.

[5] X. Chen, B. Proulx, X. Gong, and J. Zhang, "Social trust and social reciprocity based cooperative D2D communications," in Proceedings of the 14th ACM International Symposium on Mobile Ad Hoc Networking and Computing (MobiHoc '13), pp. 187-196, ACM, Bangalore, India, August 2013.

[6] I.-R. Chen, J. Guo, F. Bao, and J.-H. Cho, "Trust management in mobile ad hoc networks for bias minimization and application performance maximization," Ad Hoc Networks, vol. 19, pp. 5974, 2014.

[7] M. A. Nowak, "Five rules for the evolution of cooperation," Science, vol. 314, no. 5805, pp. 1560-1563, 2006.

[8] D. R. Amor and J. Fort, "Effects of punishment in a mobile population playing the prisoner's dilemma game," Physical Review E, vol. 84, no. 6, Article ID 066115, 2011.

[9] Z. Han, Game Theory in Wireless and Communication Networks: Theory, Models, and Applications, Cambridge University Press, Cambridge, UK, 2012.

[10] D. Peng, F. Wu, and G. Chen, "Pay as how well you do: a quality based incentive mechanism for crowdsensing," in Proceedings of the 16th ACM International Symposium on Mobile Ad Hoc Networking and Computing (MobiHoc '15), pp. 177-186, ACM, Hangzhou, China, June 2015.

[11] S. Meloni, A. Buscarino, L. Fortuna et al., "Effects of mobility in a population of prisoner's dilemma players," Physical Review E, vol. 79, no. 6, Article ID 067101, 2009. 
[12] M. A. Nowak, Evolutionary Dynamics, Harvard University Press, 2006.

[13] M. A. Nowak and R. M. May, "Evolutionary games and spatial chaos," Nature, vol. 359, no. 6398, pp. 826-829, 1992.

[14] C. P. Roca and D. Helbing, "Emergence of social cohesion in a model society of greedy, mobile individuals," Proceedings of the National Academy of Sciences of the United States of America, vol. 108, no. 28, pp. 11370-11374, 2011.

[15] C. Hauert, S. D. Monte, J. Hofbauer, and K. Sigmund, "Volunteering as Red Queen mechanism for cooperation in public goods games," Science, vol. 296, no. 5570, pp. 1129-1132, 2002.

[16] G. Wei, P. Zhu, A. V. Vasilakos, Y. Mao, J. Luo, and Y. Ling, "Cooperation dynamics on collaborative social networks of heterogeneous population," IEEE Journal on Selected Areas in Communications, vol. 31, no. 6, pp. 1135-1146, 2013.

[17] L. Song, D. Niyato, Z. Han, and E. Hossain, "Game-theoretic resource allocation methods for device-to-device communication," IEEE Wireless Communications, vol. 21, no. 3, pp. 136-144, 2014.

[18] B. Di, T. Wang, L. Song, and Z. Han, "Incentive mechanism for collaborative smartphone sensing using overlapping coalition formation games," in Proceedings of the IEEE Global Communications Conference (GLOBECOM '13), pp. 1705-1710, Atlanta, Ga, USA, December 2013.

[19] G. Wei, A. V. Vasilakos, Y. Zheng, and N. Xiong, "A gametheoretic method of fair resource allocation for cloud computing services," The Journal of Supercomputing, vol. 54, no. 2, pp. 252-269, 2010.

[20] H. Lin, D.-P. Yang, and J.-W. Shuai, "Cooperation among mobile individuals with payoff expectations in the spatial prisoner's dilemma game," Chaos, Solitons and Fractals, vol. 44, no. 1-3, pp. 153-159, 2011.

[21] J. Zhang, W.-Y. Wang, W.-B. Du, and X.-B. Cao, "Evolution of cooperation among mobile agents with heterogenous view radii," Physica A: Statistical Mechanics and its Applications, vol. 390, no. 12, pp. 2251-2257, 2011.

[22] Y.-T. Lin, H.-X. Yang, Z.-X. Wu, and B.-H. Wang, "Promotion of cooperation by aspiration-induced migration," Physica A: Statistical Mechanics and its Applications, vol. 390, no. 1, pp. 7782, 2011.

[23] F. C. Santos, M. D. Santos, and J. M. Pacheco, "Social diversity promotes the emergence of cooperation in public goods games," Nature, vol. 454, no. 7201, pp. 213-216, 2008.

[24] H. Ohtsuki, C. Hauert, E. Lieberman, and M. A. Nowak, "A simple rule for the evolution of cooperation on graphs and social networks," Nature, vol. 441, no. 7092, pp. 502-505, 2006.

[25] Z.-X. Wu and P. Holme, "Effects of strategy-migration direction and noise in the evolutionary spatial prisoner's dilemma," Physical Review E, vol. 80, no. 2, Article ID 026108, 2009.

[26] F. Wu, K. Gong, T. Zhang, G. Chen, and C. Qiao, "COMO: a game-theoretic approach for joint multirate opportunistic routing and forwarding in non-cooperative wireless networks," IEEE Transactions on Wireless Communications, vol. 14, no. 2, pp. 948-959, 2015.

[27] A. Rapoport and A. M. Chammah, Prisoner's Dilemma: A Study in Conflict and Cooperation, University of Michigan Press, Ann Arbor, Mich, USA, 1965. 

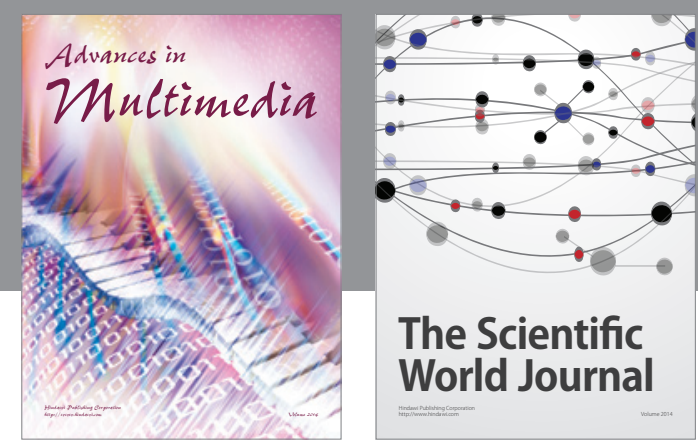

The Scientific World Journal
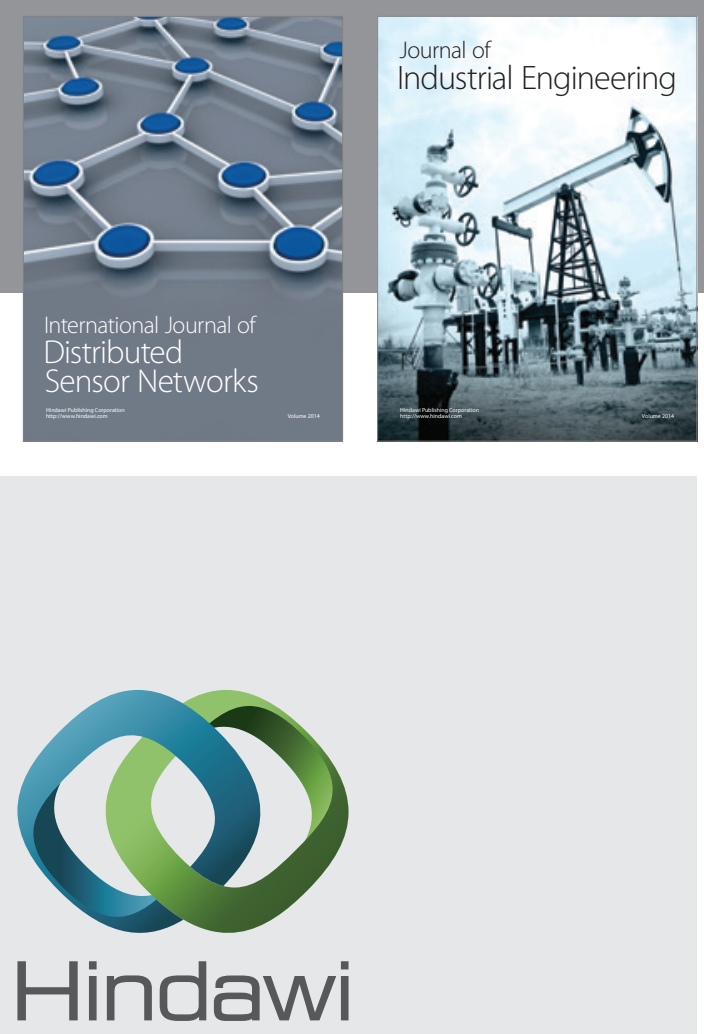

Submit your manuscripts at

http://www.hindawi.com

\section{Computer Networks} and Communications
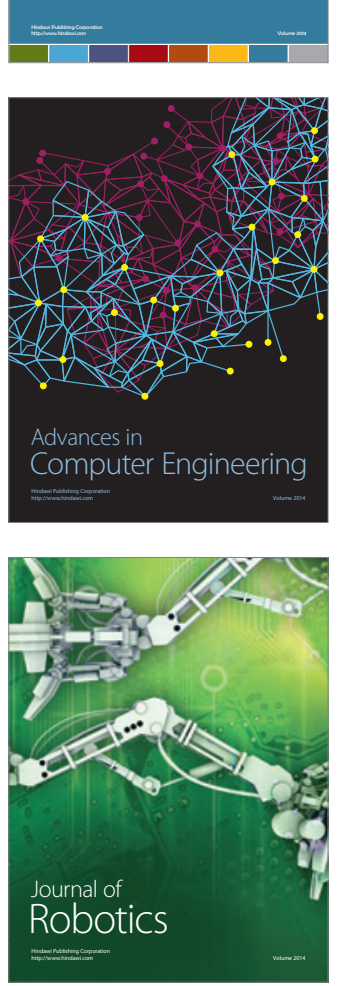
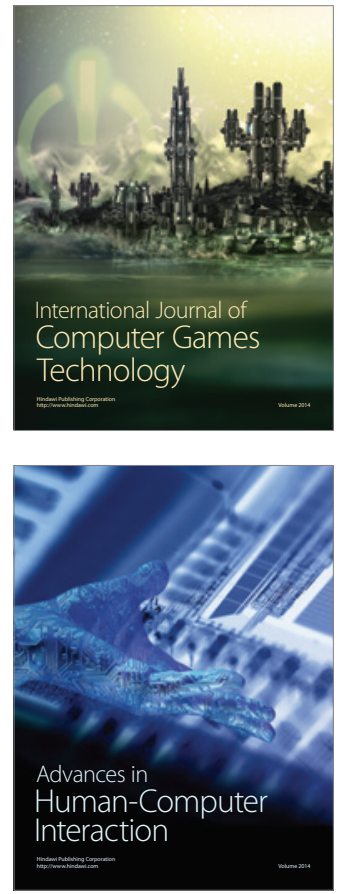
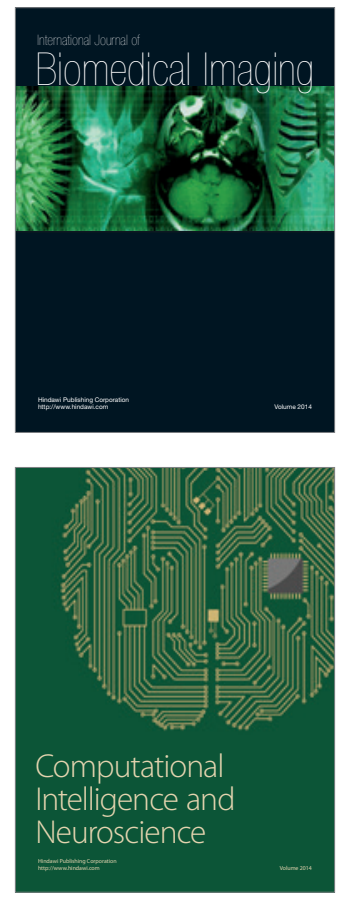
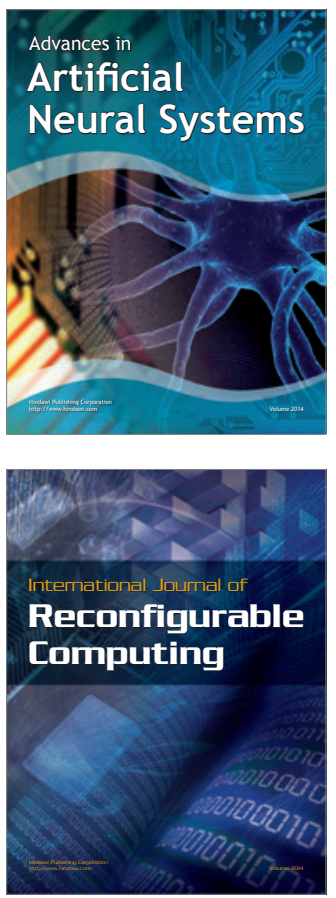
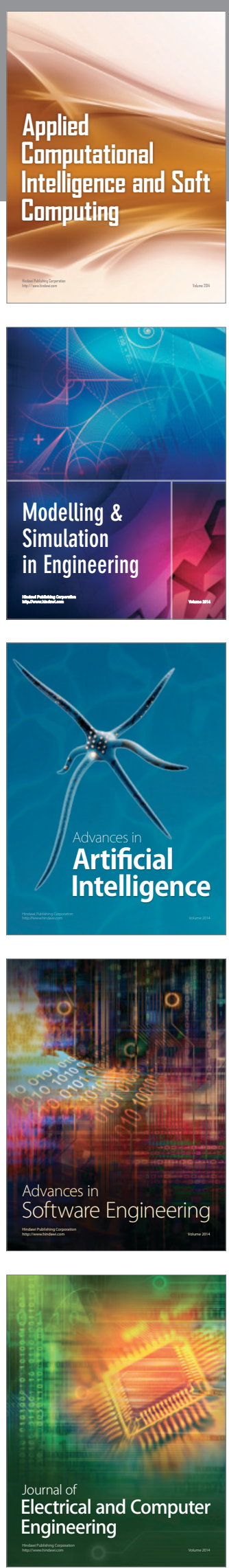